\title{
Target Specificity of Neuropeptide Y-Immunoreactive Cranial Parasympathetic Neurons
}

\author{
Gabrielle G. Leblanc and Story C. Landis \\ Department of Neurobiology, Harvard Medical School, Boston, Massachusetts 02115, and Department of Pharmacology, \\ Case Western Reserve University School of Medicine, Cleveland, Ohio 44106
}

We recently showed that neuropeptide $Y$ (NPY)-like immunoreactivity occurs in subpopulations of neurons in 3 cranial parasympathetic ganglia: the otic, sphenopalatine, and ciliary. The present work identifies the target tissues innervated by cranial parasympathetic NPY-immunoreactive neurons. Plexuses of NPY-immunoreactive fibers were observed in the parotid gland, the target of the otic ganglion, and in the intraorbital lacrimal gland and palate, targets of the sphenopalatine ganglion. NPY-immunoreactive fibers of apparent parasympathetic origin innervated glandular acini in all 3 structures and were also present around small blood vessels in the parotid and intraorbital lacrimal glands. These fibers were presumed to be parasympathetic because they were not affected by removal of the superior cervical ganglion and because their distribution was coextensive with that of vasoactive intestinal polypeptide (VIP) immunoreactivity, which we have previously shown to be colocalized with NPY in the cell bodies of otic and sphenopalatine ganglion neurons. In contrast, no NPY-immunoreactive fibers were observed in the iris or ciliary body of acutely sympathectomized rats, suggesting that NPY-immunoreactive neurons in the ciliary ganglion do not normally transport detectable levels of NPY to their terminals.

The target specificities of cranial parasympathetic NPYimmunoreactive neurons are different from those of sympathetic NPY-immunoreactive neurons. Sympathetic NPYimmunoreactive fibers innervated the iris and ciliary body, and the blood vessels but not the parenchymal cells of all the glands examined. In contrast, parasympathetic NPY-immunoreactive fibers primarily innervated glandular acini. NPYimmunoreactive neurons in the sphenopalatine ganglion displayed an additional level of specificity in their projection pattern in that they innervated only a subset of the ganglion's array of target glands: they innervated the intraorbital lacrimal gland and the seromucous glands of the palate but not the exorbital lacrimal gland or the glands of the nasal mucosa.

The finding that NPY immunoreactivity is present in the parasympathetic innervation of secretory acini in several craniofacial glands raises the possibility that NPY plays a role in the parasympathetic control of glandular secretion.

Received Mar. 9, 1987; revised June 15, 1987; accepted June 17, 1987.

This work was supported by U.S. Public Health Service Grants NS 23678, NS 02253, and NS 07112 and a grant-in-aid (84-1302) from the American Heart Association.

Correspondence should be addressed to Gabrielle G. Leblanc, Developmental Biology Center, University of California, Irvine, CA 92717.

Copyright (C) 1988 Society for Neuroscience $0270-6474 / 88 / 010146-10 \$ 02.00 / 0$
The observed overlap in the distributions of NPY- and VIP. immunoreactive fibers in these glands further suggests that NPY may interact with VIP to stimulate secretion.

Neuropeptide Y (NPY) is a 36 amino acid peptide that was originally isolated from porcine brain (Tatemoto, 1982; Tatemoto et al., 1982). NPY is structurally related to members of the pancreatic polypeptide family, but appears to be localized exclusively in central and peripheral neurons (Tatemoto, 1982; Lundberg et al., 1984c). In the periphery, NPY has previously been shown to occur in subpopulations of sympathetic neurons, where it is colocalized with norepinephrine (Jacobowitz and Olschowka, 1982; Lundberg et al., 1982a, b, 1985). The principal targets of NPY-containing sympathetic neurons are the peripheral and cerebral vasculature, consistent with functional studies that have shown that NPY is an extremely potent vasoconstrictor (Lundberg and Tatemoto, 1982; Lundberg et al., 1982b; Edvinsson et al., 1983). While in many sympathetic target structures, NPY-immunoreactive fibers are found exclusively around blood vessels (Lundberg et al., 1982a, b; Cannon et al., 1986), they innervate the parenchyma as well as the vasculature of the iris (Terenghi et al., 1983; Zhang et al., 1984; Björklund et al., 1985), heart (Gu et al., 1983, 1984; Sternini and Brecha, 1985), and urogenital tract (Lundberg et al., 1982b; Stjernquist et al., 1983; Papka et al., 1985). NPY inhibits nerve stimulation-evoked contractions in the latter 2 targets, probably via a presynaptic inhibition of neurotransmitter release (Allen et al., 1982; Lundberg et al., 1982b; Ohhashi and Jacobowitz, 1983; Stjernquist et al., 1983; Lundberg and Stj̄arne, 1984; Lundberg et al., 1984b; Franco-Cereceda et al., 1985). These findings suggest that NPY functions as a neuromodulator in the sympathetic nervous system.

Recently, we reported that NPY-immunoreactive neurons are abundant in 3 cranial parasympathetic ganglia: the otic, ciliary, and sphenopalatine (Leblanc et al., 1987). We further showed that in many of these neurons NPY is colocalized with vasoactive intestinal peptide (VIP) and/or ACh. As a first step toward understanding the role of NPY in parasympathetic neurotransmission, the present work identifies the targets of NPY-immunoreactive cranial parasympathetic neurons. We also compare the distributions of parasympathetic and sympathetic NPYimmunoreactive fibers among several craniofacial tissues.

\section{Materials and Methods}

The NPY antiserum (Amersham) was raised in rabbit against synthetic porcine NPY. The tyrosine hydroxylase (TH) antiserum, a gift from $J$. Thibault, was raised in rabbit against TH purified from rat pheochromocytoma tumors (Thibault et al., 1981). The VIP antiserum (gift from Pat Hogan, Harvard Medical School) was raised in rabbit against a 
carbodiimide conjugate of synthetic VIP (Boehringer-Mannheim) to BSA.

NPY, TH, and VIP immunoreactivities were examined with an indirect immunofluorescence method. Adult rats (Charles River, CD strain) were perfused with $4 \%$ paraformaldehyde in $0.1 \mathrm{M}$ sodium phosphate, $\mathrm{pH} \mathrm{7.3,} \mathrm{for} 10 \mathrm{~min}$. Tissues were removed and incubated in the same fixative for $1 \mathrm{hr}$, rinsed in phosphate buffer, and equilibrated for $24 \mathrm{hr}$ in $30 \%$ sucrose in phosphate buffer. Ten to $15 \mu \mathrm{m}$ cryostat sections were cut and mounted on gelatin-coated slides. Slides were rinsed with PBS and incubated overnight at room temperature in humid chambers with antisera against NPY, TH, or VIP (at dilutions of 1:500, 1:1000, and $1: 1000$, respectively) in incubation buffer $(0.01 \mathrm{~m}$ sodium phosphate, pH 7.3 containing $0.5 \mathrm{M} \mathrm{NaCl}, 0.2 \%$ Triton $\mathrm{X}-100,0.1 \%$ sodium azide, and $5 \% \mathrm{BSA})$. The sections were then rinsed with PBS and incubated for $2 \mathrm{hr}$ at room temperature with tetramethylrhodamine isothiocyanate-conjugated goat anti-rabbit IgG (Tago) diluted 1:400 in incubation buffer. After rinsing, the sections were coverslipped in glycerol: ethanol (1:1) and examined by epifluorescence using a rhodamine filter set. Staining with the NPY antiserum was abolished by preincubating the antiserum overnight at $4^{\circ} \mathrm{C}$ with $10 \mu \mathrm{M}$ synthetic porcine NPY (Bachem) before applying it to the tissue sections but was unaffected by preincubating the antiserum with $10 \mu \mathrm{M}$ peptide YY (Sigma), a peptide with structural similarities to NPY. Staining with the VIP antiserum was abolished by preincubating the antiserum with $10 \mu \mathrm{M}$ synthetic VIP (Peninsula Labs).

The following protocol was used to double-label tissue sections for (1) either NPY or VIP immunoreactivity and (2) a synaptic vesicle antigen recognized by the mousc monoclonal antibody 48 (Matthew et al., 1981). Sections were incubated as described above with either the NPY or the VIP antiserum and then with the rhodamine-conjugated goat anti-rabbit IgG. The sections were then rinsed and incubated overnight at room temperature with the 48 antibody. The sections were rinsed and incubated for $1 \mathrm{hr}$ at room temperature with a fluoresceinconjugated goat anti-mouse IgG (American Qualex), which was diluted $1: 100$ in PBS containing 50\% rat serum and $2 \%$ goat serum. The sections were then rinsed and coverslipped with $0.5 \mathrm{mg} / \mathrm{ml} p$-phenylenediamine in $50 \% 0.2 \mathrm{M}$ sodium carbonate buffer, $\mathrm{pH} 8.8,50 \%$ glycerol.

To examine the effect of sympathetic denervation on the distributions of NPY-, TH-, and VIP-immunoreactive fibers in various craniofacial tissues, uni- or bilateral removal of the superior cervical ganglion (SCG) was performed under chloral hydrate $(0.6 \mathrm{gm} / \mathrm{kg}$, s.c.) anesthesia. In most cases, only the SCG ipsilateral to the structures of interest was removed. However, bilateral removal of the SCG was required to eliminate sympathetic fibers from 2 midline structures, the septal nasal mucosa and the palate. Tissues were examined for NPY immunoreactivity $2 \mathrm{~d}$ after sympathetic denervation.

\section{Results}

Known target structures of the otic, ciliary, and sphenopalatine ganglia were examined for the presence of NPY-immunoreactive fibers. Since many of these targets receive sympathetic and sensory as well as parasympathetic innervation, it was necessary to determine the source of NPY-immunoreactive fibers in these structures. It seemed unlikely that NPY immunoreactivity would be present in sensory fibers: it has been reported previously that NPY immunoreactivity is not detectable in cranial or dorsal root sensory ganglion neurons (Lundberg et al., 1983), and we observed only occasional immunoreactive neurons in the trigeminal ganglion with the commercial NPY antiserum used in the present study. However, NPY-immunoreactive neurons are abundant in the SCG (Lundberg et al., 1982b), the major source of sympathetic innervation to cranial target tissues. The following methods were used to determine whether NPY-immunoreactive fibers in various tissues were of sympathetic or parasympathetic origin. First, we compared the distribution of NPY-immunoreactive fibers in each tissue with the distribution of sympathetic fibers, which were visualized by staining with an antiserum to $\mathrm{TH}$. Second, we examined the effect of bilateral removal of the SCG on the distribution of NPY-immunoreactive fibers in each tissue. Finally in target tissues of the otic and sphenopalatine ganglia, the distribution of parasympathetic fibers was determined by staining for VIP. VIP is present in virtually all otic and sphenopalatine neurons in the rat (Lundberg et al., 1984a; Leblanc et al., 1987), while only rare neurons in the rat SCG contain VIP (Hökfelt et al., 1977). VIP-immunoreactive fibers have been observed in all otic and sphenopalatine ganglion target tissues examined, and in all cases these fibers are eliminated by parasympathetic denervation (Uddman et al., 1980a-c; Lundberg et al., 1981; Brodin et al., 1983; Butler et al., 1984; Dartt et al., 1984; Nikkinen et al., 1984). Thus, VIP provided a marker for otic and sphenopalatine terminals in their respective target tissues with which the distribution of NPY-immunoreactive fibers was then compared.

\section{Otic ganglion}

The otic ganglion innervates the parotid gland (Warwick and Williams, 1973; Brodin et al., 1983; Sharkey and Templeton, 1984). The distribution of parasympathetic and sympathetic fibers in the parotid was assessed by staining for VIP and TH, respectively. We observed a plexus of VIP-immunoreactive fibers surrounding parotid acini (Fig. 1c). TH-immunoreactive fibers were also present around parotid acini (Fig. 1e). Removal of the ipsilateral SCG eliminated the TH-immunoreactive fibers (Fig. 1 $f$ ) but, as expected, had no effect on the density of the VIP-immunoreactive fibers (Fig. $1 d$ ). These observations are consistent with previous evidence that parotid acini are dually innervated by parasympathetic and sympathetic neurons (e.g., Hand, 1972).

Consistent with our previous finding that most neurons in the otic ganglion contain NPY, we observed a plexus of NPY-immunoreactive fibers surrounding secretory acini in the parotid (Fig. 1a). Sympathetic denervation had no appreciable effect on the density of NPY-immunoreactive terminals in the parenchyma of the parotid gland (Fig. $1 b$ ). Consistent with this immunocytochemical observation, we found that sympathectomy had little effect on the levels of NPY immunoreactivity measured in the gland by radioimmunoassay: levels of NPY immunoreactivity in sympathetically denervated parotid glands were $92.2 \pm 5.4 \%$ (mean \pm SEM for 3 animals) of those in contralateral control glands. Finally, we found that the distribution and density of NPY-immunoreactive fibers in the parotid glands of sympathectomized rats was similar to that of VIPimmunoreactive fibers (Fig. $1, b, d$ ). These findings support the conclusion that most or all of the NPY-immunoreactive fibers that innervate secretory acini in the parotid gland arise from the otic ganglion.

The blood vessels as well as the secretory acini of the parotid gland are innervated by autonomic nerve fibers. NPY, TH, and VIP immunoreactivities were each present in nerve fibers around parotid blood vessels (Fig. 2). Both the NPY-immunoreactive fibers and the $\mathrm{TH}$-immunoreactive fibers were more prominent around the larger-diameter blood vessels in the connective tissue capsule and septa of the gland than around the smaller intralobular blood vessels. Removal of the SCG eliminated all of the perivascular TH-immunoreactive fibers in the parotid gland, and most of the NPY-immunoreactive fibers that were present around larger blood vessels. However, many NPY- and VIPimmunoreactive fibers remained around the smaller vessels after sympathectomy (Fig. 2, c, d). These results suggest that many of the NPY-immunoreactive fibers that innervate small blood vessels in the parotid gland are parasympathetic.

We have shown previously that NPY and VIP are colocalized 


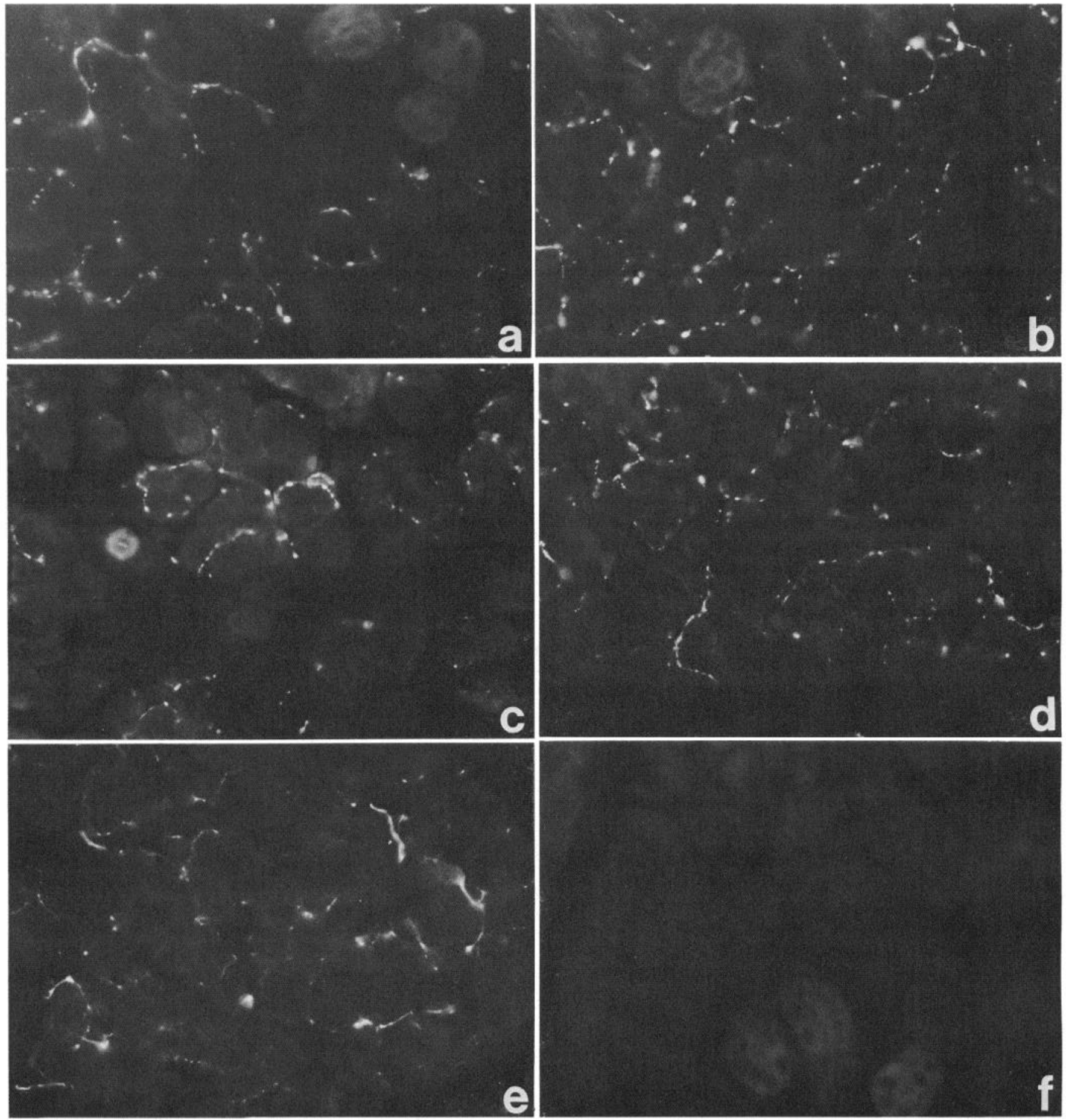

Figure 1. Immunofluorescence micrographs of sections of parotid glands from control $(a, c, e)$ and sympathectomized $(b, d, f)$ rats stained for NPY $(a, b)$, VIP $(c, d)$, or TH $(e, f)$. Plexuses of NPY-immunoreactive fibers $(a)$, VIP-immunoreactive fibers $(c)$, and TH-immunoreactive fibers $(e)$ surround secretory acini in the parotid glands of control rats. Removal of the ipsilateral SCG eliminates the TH-immunoreactive fibers $(f)$ but does not affect the density of the NPY-immunoreactive fibers $(b)$ or VIP-immunoreactive fibers $(d) . \times 420$.

in otic ganglion neurons (Leblanc et al., 1987). Since the distribution and density of NPY-immunoreactive fibers in the parotid glands of sympathectomized rats was similar to that of VIP-immunoreactive fibers, it seemed likely that the 2 peptides are also colocalized in otic nerve terminals in the parotid. To investigate this possibility further, we double-stained sections of the parotid glands of sympathectomized rats with (1) anti- serum to either NPY or VIP, and (2) the monoclonal antibody 48 , which recognizes a synaptic vesicle protein (Matthew et al., 1981). The 48 antibody labels both sympathetic and parasympathetic, but not sensory (Jane Dodd, personal communication), nerve terminals. Virtually all of the terminal profiles labeled with this antibody in sections of sympathetically denervated parotid glands also contained NPY immunoreactivity and VIP 


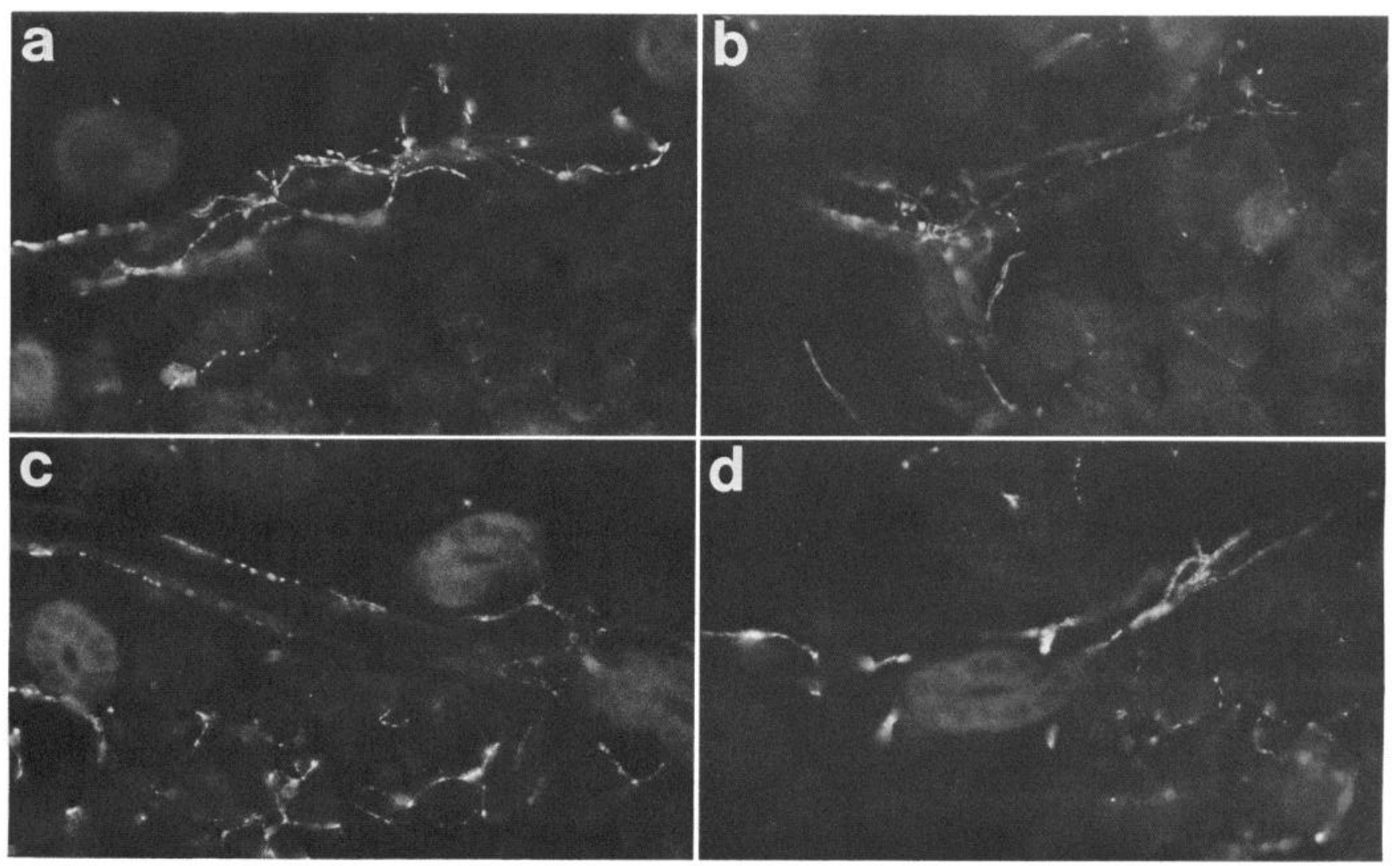

Figure 2. Immunofluorescence micrographs of sections of the parotid glands of control $(a, b)$ and sympathectomized $(c, d)$ rats stained for NPY $(a, c)$, TH $(b)$, and VIP $(d)$. Both NPY-immunoreactive fibers $(a)$ and TH-immunoreactive fibers $(b)$ are present around blood vessels in the parotid glands of control rats. Removal of the ipsilateral SCG eliminates the TH-immunoreactive innervation of parotid blood vessels (not shown), but many NPY-immunoreactive fibers remain $(c)$. VIP-immunoreactive fibers are also found around parotid blood vessels in both control (not shown) and sympathectomized $(d)$ rats. $\times 365$.

immunoreactivity, respectively (Fig. 3). This result is consistent with the conclusion that NPY and VIP coexist in otic nerve terminals in the parotid gland.

\section{Sphenopalatine ganglion}

The sphenopalatine ganglion innervates several different target structures, including the intra- and exorbital lacrimal glands, the palate, and the nasal mucosa (Ruskell, 1971; Warwick and Williams, 1973; Uddman, 1980a, c). The distribution of sphenopalatine terminals in these structures was assessed by staining for VIP. Consistent with previous reports (Uddman et al., 1980a, c; Dartt et al., 1984; Nikkinen et al., 1984), we observed VIPimmunoreactive fibers around both glandular acini and blood vessels in the nasal mucosa and lacrimal glands. A plexus of VIP-immunoreactive fibers was also observed surrounding the seromucous glands of the palate.

NPY-immunoreactive fibers were present around glandular acini in the palate and intraorbital lacrimal gland; their distribution and density was similar to that of the VIP-immunoreactive fibers. No $\mathrm{TH}$-immunoreactive fibers were seen in the parenchyma of these targets, suggesting the absence of significant sympathetic innervation. Consistent with this supposition, we found that sympathectomy had no effect on the density of the NPY-immunoreactive fibers in these targets. These findings suggest that the NPY-immunoreactive fibers which innervate glandular acini in the palate and intraorbital lacrimal gland arise from the sphenopalatine ganglion.
Scattered NPY- and TH-immunoreactive fibers were seen around glandular acini in the nasal mucosa. Only occasional NPY-immunoreactive fibers (and no TH-immunoreactive fibers) remained after sympathectomy. In contrast, dense plexuses of VIP-immunoreactive fibers were present around glandular acini in the nasal mucosa of both control and sympathectomized rats. Similarly, while numerous VIP-immunoreactive fibers were present around acini in the exorbital lacrimal gland, no NPYimmunoreactive fibers were present in the parenchyma of this gland.

Our observations concerning the distributions of NPY- and VIP-immunoreactive fibers around glandular acini in targets of the sphenopalatine ganglion in sympathectomized rats are summarized in Figure 4. In 2 targets of the sphenopalatine ganglion, the palate and intraorbital lacrimal gland, glandular acini were innervated by NPY-immunoreactive fibers (Fig. 4, $a, c$ ) whose distribution matched that of VIP-immunoreactive fibers (Fig. $4, b, d)$. In contrast, in 2 other sphenopalatine targets, the nasal mucosa and the exorbital lacrimal gland, many VIP-immunoreactive fibers (Fig. $4, f, h$ ) but few or no NPY-immunoreactive fibers (Fig. $4, e, g$ ) were present around glandular acini. These results are consistent with the conclusion that NPY-immunoreactive neurons in the sphenopalatine innervate secretory acini in the intraorbital lacrimal gland and palate but not the nasal mucosa or exorbital lacrimal gland.

NPY-immunoreactive nerve fibers were observed around blood vessels in all of the sphenopalatine targets examined, as 

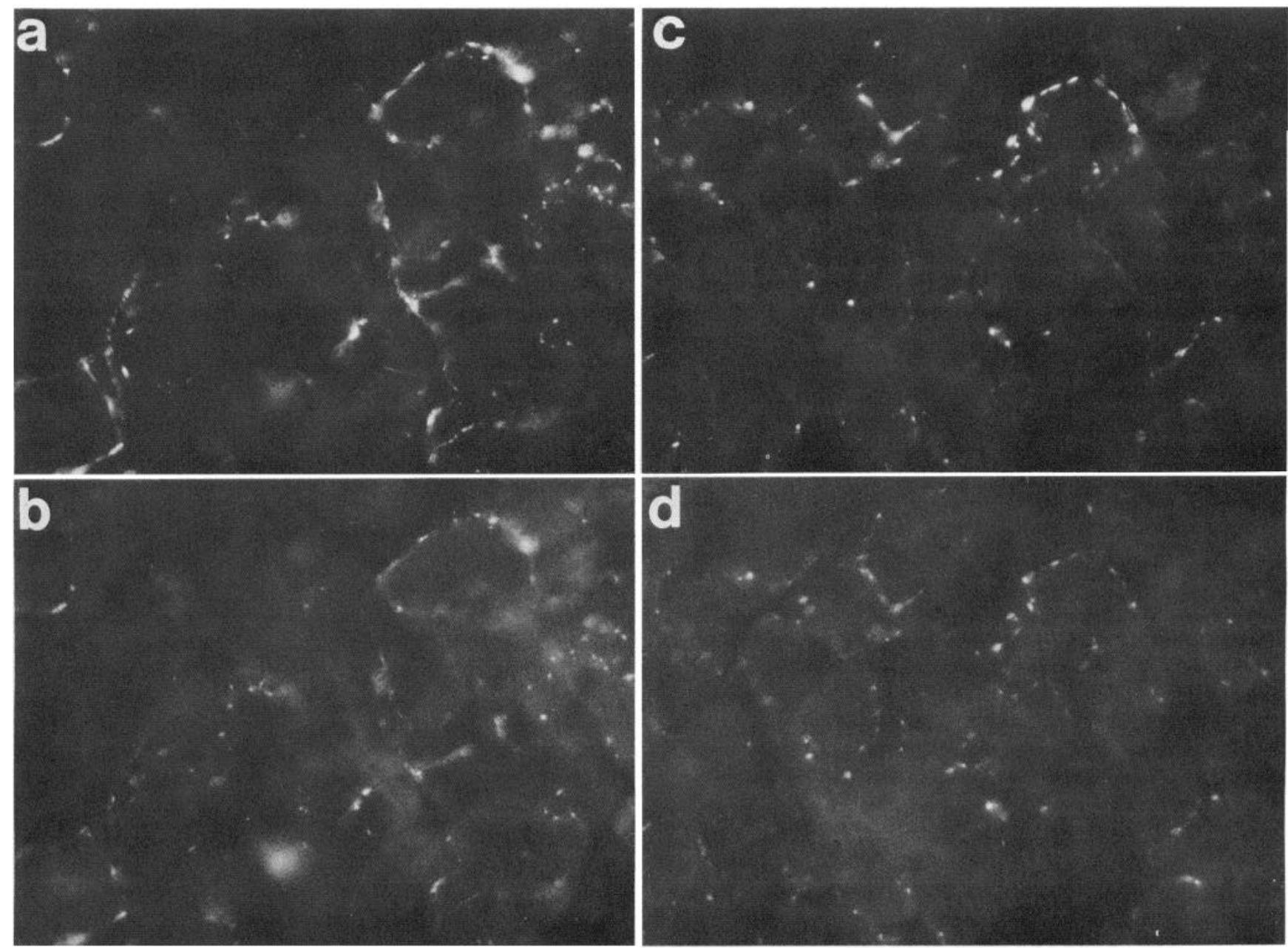

Figure 3. Immunofluorescence micrographs of sections of the parotid gland of a sympathectomized rat double-stained for NPY (a) and synaptic vesicle antigen $(b)$, or VIP $(c)$ and synaptic vesicle antigen $(d)$. NPY and VIP immunoreactivities were visualized using primary antisera raised in rabbit followed by a rhodamine-coupled second antibody, while synaptic vesicle antigen was visualized using a mouse monoclonal antibody followed by a fluorescein-coupled second antibody. Virtually all of the fibers labeled with the antibody to synaptic vesicle antigen $(b, d)$ are also labeled with both the NPY antiserum $(a)$ and VIP antiserum $(c)$, respectively. $\times 500$.

were TH- and VIP-immunoreactive fibers. All of the perivascular TH-immunoreactive fibers in these targets disappeared after sympathectomy, while the VIP-immunoreactive fibers were unaffected. Sympathectomy eliminated all of the perivascular NPY-immunoreactive fibers in the exorbital lacrimal gland and nasal mucosa and most of those in the palate. In contrast, sympathectomy caused only a partial reduction in the number of perivascular NPY-immunoreactive fibers in the intraorbital lacrimal gland (Fig. 5). As was true for the parotid, the larger, intracapsular and intraseptal blood vessels were more completely depleted of NPY-immunoreactive fibers after sympathectomy than were the smaller, intralobular vessels. Thus, while most or all of the perivascular NPY-immunoreactive fibers in the exorbital lacrimal gland, nasal mucosa, and palate are sympathetic in origin, many of the NPY-immunoreactive fibers that innervate blood vessels in the intraorbital lacrimal gland appear to be parasympathetic.

\section{Ciliary ganglion}

The ciliary ganglion innervates the iris and ciliary body. Our observations concerning the distribution of NPY-immunoreactive fibers in these structures are in agreement with previous reports (Terenghi et al., 1983; Zhang et al., 1984; Björklund et al., 1985). We observed a rich plexus of NPY-immunoreactive fibers in the iris, and numerous NPY-immunoreactive fibers in the ciliary processes. TH-immunoreactive fibers were also present in both structures and were considerably more numerous than NPY-immunoreactive fibers in the iris. Two days after removal of the ipsilateral SCG, only occasional, weakly immunofluorescent NPY-immunoreactive fibers were seen in the iris and ciliary processes. This result indicates that virtually all of the NPY-immunoreactive fibers normally present in the iris are sympathetic. We have previously found that $25-30 \%$ of the neurons in the ciliary ganglion contain NPY immunoreactivity. However, the few, weakly NPY-immunoreactive fibers seen in the irides of sympathectomized rats constituted considerably less than a quarter of the total parasympathetic plexus present in the iris, as assessed by staining the irides of acutely sympathectomized rats for synaptic vesicle antigen or acetylcholinesterase activity. It is conceivable that the NPY-immunoreactive neurons in the rat ciliary project to targets other than the iris and ciliary body. However, it has been shown in mouse, at least, that ciliary nerve terminals labeled anterogradely by application of HRP to the ganglion are found exclusively in the 


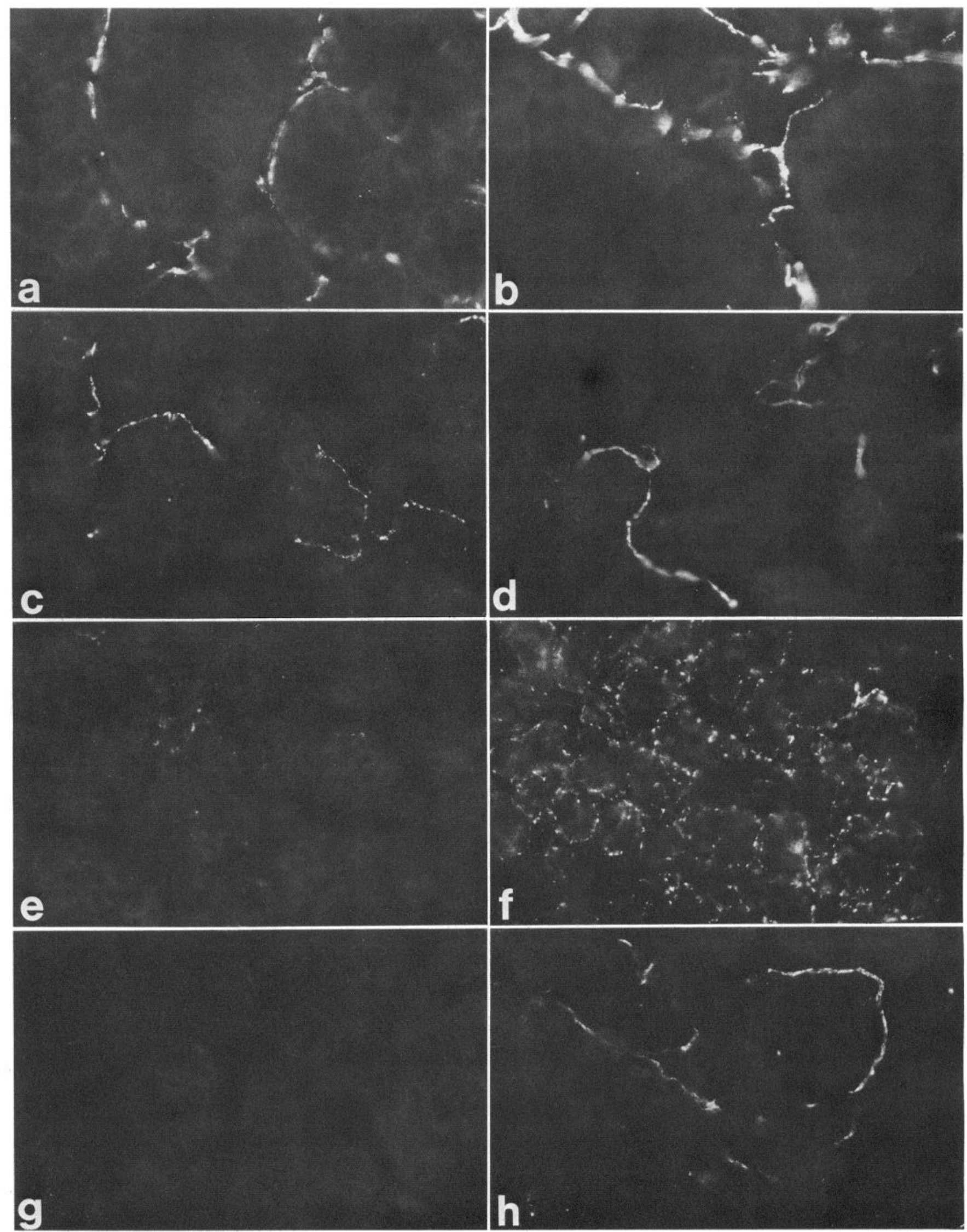

Figure 4. Immunofluorescence micrographs of sections of 4 target structures of the sphenopalatine ganglion stained for NPY $(a, c, e, g)$ or VIP $(b, d, f, h)$. All sections are taken from sympathectomized rats. $a$ and $b$, Seromucous glands of the palate. $\times 380 . c$ and $d$, Intraorbital lacrimal gland. $\times 365 . e$ and $f$, Nasal mucosa. $\times 400 . g$ and $h$, Exorbital lacrimal gland. $\times 305$. Plexuses of VIP-immunoreactive fibers surround secretory acini in all 4 structures $(b, d, f, h)$, while NPY-immunoreactive fibers innervate secretory acini in the palate $(a)$ and intraorbital lacrimal gland $(c)$, but not in the nasal mucosa $(e)$ or exorbital lacrimal gland $(g)$. 
iris and ciliary body (Jackson, 1986). Hence, the present results suggest that most NPY-immunoreactive neurons in the ciliary do not normally transport immunocytochemically detectable levels of NPY immunoreactivity to their terminals.

\section{Discussion}

We recently showed that NPY immunoreactivity is present in subpopulations of neurons in 3 cranial parasympathetic ganglia - the otic, sphenopalatine, and ciliary (Leblanc et al., 1987). The present study describes the projections of NPY-immunoreactive cranial parasympathetic neurons. We have presented evidence here that NPY-immunoreactive neurons in the otic ganglion project to the parotid gland and that those in the sphenopalatine ganglion project to the intraorbital lacrimal gland and palate. NPY-immunoreactive fibers of apparent parasympathetic origin innervate secretory acini in all of these glands and are also present around small, intralobular blood vessels in the parotid and intraorbital lacrimal glands. While definitive proof that these fibers are parasympathetic requires either removal of the appropriate parasympathetic ganglia or retrograde labeling combined with immunocytochemistry, the following observations are consistent with this conclusion. First, the fibers are unaffected by removal of the SCG, the major (and probably sole) source of sympathetic innervation to craniofacial tissues. Second, in sympathectomized rats, the distribution of NPYimmunoreactive fibers in the parotid, intraorbital lacrimal gland, and palate overlaps with that of VIP-immunoreactive fibers. The VIP-immunoreactive fibers present in the parotid and intraorbital lacrimal gland have previously been shown to disappear after parasympathetic denervation (Uddman et al., 1980a; Brodin et al., 1983; Butler et al., 1984), although the palate has not yet been examined in this respect.

We had observed previously that $25-30 \%$ of the neurons in the ciliary ganglion contain NPY immunoreactivity (Leblanc et al., 1987). While the NPY-immunoreactive neurons in the ciliary ganglion presumably innervate the iris and/or ciliary body, NPY immunoreactivity is not detectable in parasympathetic terminals in these structures after acute sympathectomy. The observation that NPY immunoreactivity is detectable in the cell bodies but not the terminals of ciliary neurons is the converse of the more commonly encountered situation, in which a peptide found in the terminals of a particular population of neurons is undetectable in their cell bodies unless colchicine treatment is used to block axonal transport. However, there is some evidence that the distribution of substance P in SCG neurons is similar to that of NPY in ciliary neurons: substance $\mathrm{P}$ is detectable by radioimmunoassay in the SCG but is undetectable in SCG terminals in the pineal and iris (Kessler et al., 1983). The present findings and those of Kessler and coworkers highlight the caution that must be used in assuming that a putative neurotransmitter molecule detected in the cell bodies of a given population of neurons is necessarily transported to their terminals. Our observations also raise the possibility that the NPY-immunoreactive material present in ciliary neuron cell bodies is a structurally modified form of either NPY or its precursor protein which cannot be incorporated into secretory vesicles. Alternatively, the NPY-immunoreactive material in ciliary cell bodies may undergo structural modifications prior to reaching ciliary terminals such that it is no longer detectable by the NPY antiserum. Finally, ciliary neurons may lack the mechanisms required for packaging and transport of NPY. Interestingly, Björklund and coworkers (1985) reported that NPY immunoreactivity
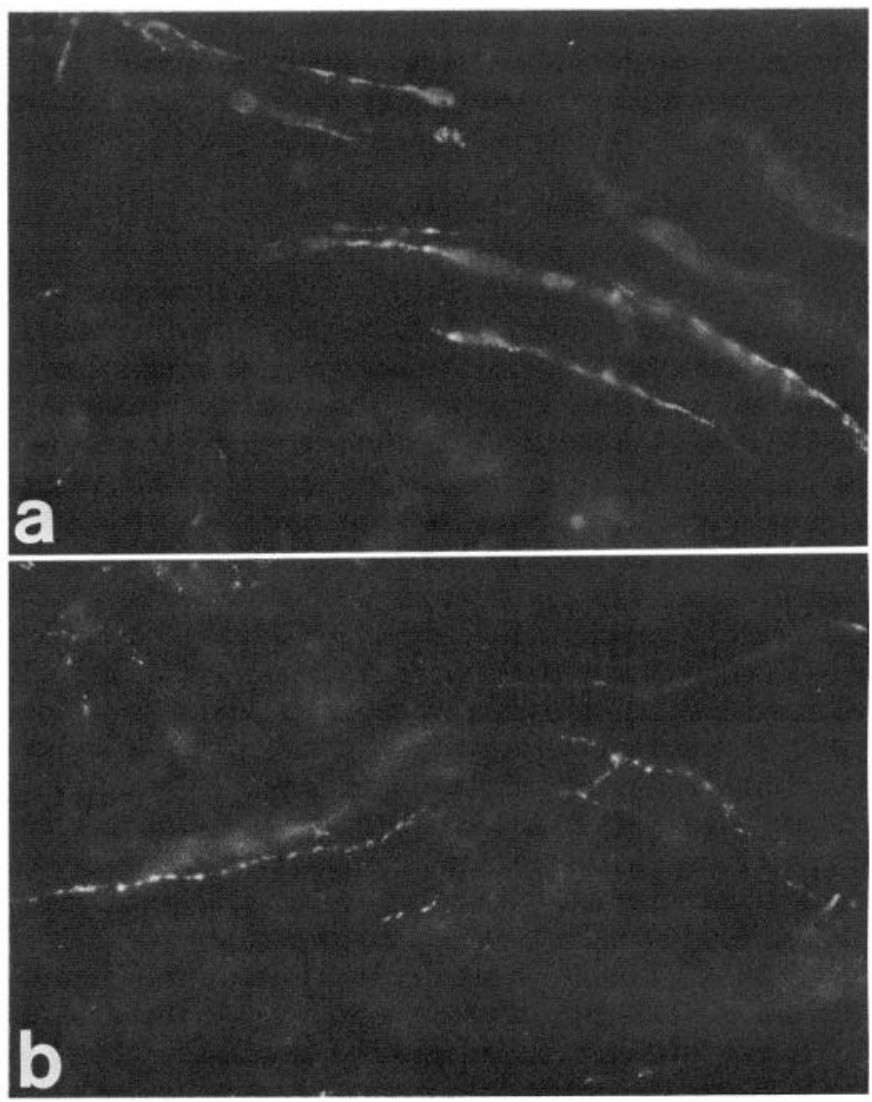

Figure 5. Immunofluorescence micrographs of sections of the intraorbital lacrimal glands of control $(a)$ and sympathectomized $(b)$ rats stained for NPY. Many NPY-immunoreactive fibers are present around blood vessels in the intraorbital lacrimal gland both before $(a)$ and after $(b)$ removal of the ipsilateral SCG. $\times 360$.

does become detectable in ciliary terminals in the iris after longterm sympathectomy. They interpreted this observation as indicating that ciliary neurons change their neurotransmitter phenotype in response to sympathetic denervation of their target. Our finding that NPY is easily detectable in the cell bodies of ciliary neurons of control rats suggests that the appearance of NPY immunoreactivity in ciliary terminals subsequent to sympathetic denervation does not involve a de novo induction of NPY expression in ciliary neurons. However, it is possible that sympathectomy does induce qualitative changes in the processing of the NPY precursor protein or its primary mRNA transcript, or in NPY packaging and transport.

NPY has previously been shown to be widely distributed in the sympathetic nervous system, where it is colocalized with norepinephrine (Jacobowitz and Olschowka, 1982; Lundberg et al., 1982a, b, 1985). Studies aimed at exploring the biological actions of NPY have consequently focused on target tissues of sympathetic NPY-immunoreactive neurons, such as the vasculature, heart, and urogenital system. Like norepinephrine, NPY has a direct vasoconstrictive action on both peripheral and central vascular beds; NPY also potentiates norepinephrine-induced vasoconstriction (Lundberg and Tatemoto, 1982; Lundberg et al., 1982b; Edvinsson et al., 1983). Other actions that have been reported for NPY include inhibition of nerve stimulation-evoked contractions of the uterus, bladder, heart, and vas deferens (Allen et al., 1982; Lundberg et al., 1982b; Ohhashi and Jacobowitz, 1983; Stjernquist et al., 1983; Lundberg et al., 
1984b). In the latter 2 cases, there is evidence that this effect is due to a presynaptic inhibition of norepinephrine release (Lundberg and Stj̄arne, 1984; Franco-Cereceda et al., 1985). These findings suggest that a major function of NPY in the periphery is to modulate noradrenergic sympathetic neurotransmission to smooth and cardiac muscle tissues.

The present finding that NPY immunoreactivity is present in parasympathetic fibers which innervate secretory acini in the parotid gland, intraorbital lacrimal gland, and seromucous glands of the palate raises the possibility that NPY also plays a role in the parasympathetic regulation of glandular secretion. Several effects of NPY on glandular function have, in fact, been reported. NPY has been found to potentiate isoproterenol-, thyroid stimulating hormone-, and VIP-stimulated thyroid hormone secretion from the thyroid gland (Grunditz et al., 1984). NPY has also bcen shown to stimulate the release of luteinizing hormone and growth hormone from the pituitary in vitro (McDonald et al., 1985). Finally, NPY has been found to inhibit glucosestimulated insulin release from the pancreas in vivo and in vitro (Moltz and McDonald, 1985).

We have shown that the distribution of NPY in the parotid, intraorbital lacrimal gland, and palate overlaps with that of VIP. This finding, together with our previous observation that NPY and VIP immunoreactivities are colocalized in otic and sphenopalatine cell bodies, is consistent with the idea that NPY and VIP coexist in parasympathetic nerve terminals in the parotid gland, intraorbital lacrimal gland, and palate. We provided further evidence for this supposition with respect to otic terminals in the parotid by showing that the distributions of NPY and VIP are each coextensive with that of synaptic vesicle antigen in the parotid glands of sympathectomized rats. It is likely that in many otic and sphenopalatine terminals NPY coexists with $\mathrm{ACh}$ as well as VIP, since we have shown previously that NPYimmunoreactive cells bodies in the otic and sphenopalatine ganglia contain immunoreactivity for choline acetyltransferase (Leblanc et al., 1987). Costored neurotransmitter molecules have in many cases been found to exert complementary physiological effects. Hence, just as in sympathetic neurotransmission NPY may interact with norepinephrine to regulate smooth and cardiac muscle tone, in parasympathetic neurotransmission NPY may interact with ACh and VIP to regulate glandular secretion. Indeed, as indicated above, it has been shown that NPY is able to potentiate VIP stimulation of thyroid hormone secretion (Grunditz et al., 1984). Both ACh and VIP stimulate secretion in many glands, including the rat parotid (Inoue and Kanno, 1982; Ekström et al., 1983; Gallacher, 1983) and exorbital lacrimal glands (Dartt et al., 1984). It would therefore be of interest to examine the effect of NPY on basal and ACh- or VIP-stimulated secretion in the glands that have been shown here to receive NPY-immunoreactive innervation.

NPY-immunoreactive fibers innervate blood vessels in all of the structures examined here. Removal of the SCG eliminates the NPY-immunoreactive innervation of blood vessels in the iris, exorbital lacrimal gland, palate and nasal mucosa, indicating that these fibers are sympathetic in origin. In the parotid and intraorbital lacrimal gland, however, many NPY-immunoreactive fibers remain around blood vessels after sympathectomy. These fibers are particularly prominent around smallerdiameter, intralobular blood vessels. These observations suggest that many of the perivascular NPY-immunoreactive fibers in the parotid and intraorbital lacrimal glands are parasympathetic. The functional significance of NPY in the parasympathetic innervation of blood vessels in the parotid and intraorbital lacrimal glands is not clear: NPY is a vasoconstrictor, but parasympathetic nerve stimulation elicits vasodilation in most glands, including the parotid (Andersson et al., 1982). Similarly, both VIP and ACh, which we have found to be costored with NPY in otic and sphenopalatine neurons, are vasodilators (Said and Mutt, 1970; Shimizu and Taira, 1979; Bloom and Edwards, 1980; Lundberg et al., 1980; Edvinsson et al., 1982). It is possible that the NPY-immunoreactive fibers we observed around blood vessels in the parotid and intraorbital lacrimal glands of sympathectomized rats are preterminal axons rather than neuroeffector junctions or that the small blood vessels in these glands are insensitive to NPY. Alternatively, the finding that concentrations of NPY lower than those required to cause vasoconstriction actually inhibit the release of norepinephrine from the sympathetic innervation of several vascular beds (Pernow et al., 1986) suggests a mechanism whereby the release of NPY from parasympathetic nerve terminals could result in vasodilation.

Previous work has indicated that NPY-containing sympathetic neurons exhibit selectivity in the types of target tissues they innervate. NPY is present in only a subpopulation of sympathetic neurons (Lundberg et al., 1982b), and there is evidence that NPY-containing sympathetic neurons project preferentially to a subset of sympathetic target tissues. Thus, in several structures that receive sympathetic innervation to both their vascular and parenchymal compartments, NPY is present only in the vascular innervation (Lundberg et al., 1982a, b; Cannon et al., 1986). In the present study, for instance, we observed that NPY is present in the sympathetic fibers that innervate blood vessels in the parotid gland but is absent from the sympathetic fibers innervating secretory acini in the same structure.

We found that there is very little overlap in the projections of cranial parasympathetic and sympathetic NPY-immunoreactive neurons among the structures examined here. Sympathetic NPY-immunoreactive fibers arising from the SCG innervate blood vessels in all of the structures examined, and also innervate parenchymal cells in the iris and ciliary body. In contrast, parasympathetic NPY-immunorcactive fibers primarily innervate secretory acini in the parotid gland, intraorbital lacrimal gland, and palate. The lack of overlap in the distribution of parasympathetic and sympathetic NPY-immunoreactive terminals is consistent with the generalization that parasympathetic and sympathetic neurons evoke different physiological responses in a given target tissue: one would not expect both the sympathetic and parasympathetic innervation of a particular tissue to share a common neurotransmitter or neuromodulator. Our observations further suggest that sympathetic and parasympathetic NPY-immunoreactive neurons differ in their target specificities. While sympathetic NPY-immunoreactive neurons project primarily to blood vessels and other smooth muscle targets such as the iris, vas deferens, and uterus, parasympathetic NPY-immunoreactive neurons primarily innervate glandular acini.

A sccond level of specificity was evident in the projections of NPY-immunoreactive neurons of the sphenopalatine ganglion. We found that NPY-immunoreactive neurons in the sphenopalatine ganglion innervate only a subset of the ganglion's array of target glands: they innervate the palate and intraorbital lacrimal gland but not the exorbital lacrimal gland or nasal mucosa. Thus, these neurons exhibit specificity with respect to the location as well as the type of target tissue they innervate. The 
developmental mechanisms that give rise to the target specificity evident in the projections of peripheral NPY-immunoreactive neurons remain to be elucidated.

\section{References}

Allen, J. M., T. E. Adrian, K. Tatemoto, J. M. Polak, J. Hughes, and S. R. Bloom (1982) Two novel related peptides, neuropeptide $Y$ (NPY) and peptide YY (PYY) inhibit the contraction of the electrically stimulated mouse vas deferens. Neuropeptides 3: 71-77.

Andersson, P.-O., S. R. Bloom, and A. V. Edwards (1982) Parotid responses to stimulation of the parasympathetic innervation in bursts in weaned lambs. J. Physiol. (Lond.) 330: 163-174.

Björklund, H., T. Hökfelt, M. Goldstein, L. Terenius, and L. Olson (1985) Appearance of the noradrenergic markers tyrosine hydroxylase and neuropeptide $\mathrm{Y}$ in cholinergic nerves of the iris following sympathectomy. J. Neurosci. 5: 1633-1643.

Bloom, S. R., and A. V. Edwards (1980) Vasoactive intestinal peptide in relation to atropine resistant vasodilation in the submaxillary gland of the cat. J. Physiol. (Lond.) 300: 41-53.

Brodin, E., R. Ekman, J. Ekström, R. Håkanson, and F. Sundler (1983) Effect of denervation on substance $P$ and vasoactive intestinal peptide in rat salivary glands. J. Physiol. (Lond.) 348: 67P.

Butler, J. M., G. L. Ruskell, D. F. Cole, W. G. Unger, S. Q. Zhang, M. A. Blank, G. P. McGregor, and S. R. Bloom (1984) Effects of VIIth (facial) nerve degeneration on vasoactive intestinal polypeptide and substance $P$ levels in ocular and orbital tissues of the rabbit. Exp. Eye Res. 39: 523-532.

Cannon, B., J. Nedergaard, J. M. Lundberg, T. Hökfelt, L. Terenius, and M. Goldstein (1986) 'Neuropeptide tyrosine' (NPY) is co-stored with noradrenaline in vascular but not in parenchymal sympathetic nerves of brown adipose tissue. Exp. Cell Res. 164: 546-550.

Dartt, D. A., A. K. Baker, C. Vaillant, and P.E. Rose (1984) Vasoactive intestinal polypeptide stimulation of protein secretion from rat lacrimal gland acini. Am. J. Physiol. 247: G502-G509.

Edvinsson, L., J. McCulloch, and R. Uddman (1982) Feline cerebral veins and arteries: Comparison of autonomic innervation and vasomotor responses. J. Physiol. (Lond.) 325: 161-173.

Edvinsson, L., P. Emson, J. McCulloch, K. Tatemoto, and R. Uddman (1983) Neuropeptide $Y$ : Cerebrovascular innervation and vasomotor effects in the cat. Neurosci. Lett. 43: 79-84.

Ekström, J., B. Månsson, and G. Tobin (1983) Vasoactive intestinal peptide evoked secretion of fluid and protein from rat salivary glands and the development of supersensitivity. Acta Physiol. Scand. 119: 169-175.

Franco-Cereceda, A., J. M. Lundberg, and C. Dahlof (1985) Neuropeptide $y$ and sympathetic control of heart contractility and coronary vascular tone. Acta Physiol. Scand. 129: 361-370.

Gallacher, D. V. (1983) Substance $P$ is a functional neurotransmitter in the rat parotid gland. J. Physiol. (Lond.) 342: 483-498.

Grunditz, T., R. IIåkanson, C. Rerup, F. Sundler, and R. Uddman (1984) Neuropeptide $Y$ in the thyroid gland: Neuronal localization and enhancement of stimulated thyroid hormone secretion. Endocrinology 115: 1537-1542.

Gu, J., T. E. Adrian, K. Tatemoto, J. M. Polak, J. M. Allen, and S. R. Bloom (1983) Neuropeptide tyrosine (NPY)-A major cardiac neuropeptide. Lancet $1: 1008$.

Gu, J., J. M. Polak, J. M. Allen, W. M. Huang, M. N. Sheppard, K. Tatemoto, and S. R. Bloom (1984) High concentrations of a novel peptide, neuropeptide $Y$, in the innervation of mouse and rat heart. J. Histochem. Cytochem. 32: 467-472.

Hand, A. R. (1972) Adrenergic and cholinergic terminals in the rat parotid gland. Electron microscopic observations on permanganatefixed glands. Anat. Rec. 173: 131-140.

IIökfelt, T., L.-G. Elfvin, M. Schultzberg, K. Fuxe, S. I. Said, V. Mutt, and M. Goldstein (1977) Immunohistochemical evidence of vasoactive intestinal polypeptide-containing neurons and nerve fibers in sympathetic ganglia. Neuroscience 2: 885-896.

Inoue, Y., and T. Kanno (1982) Secretory effects of vasoactive intestinal polypeptide (VIP), adrenaline, and carbachol on isolated lobules of the rat parotid gland. Biomed. Res. 3: 384-389.

Jackson, P. (1986) Innervation of the iris by individual parasympathetic axons in the adult mouse. J. Physiol. (Lond.) 378: 485-495.

Jacobowitz, D. M. and J. A. Olschowka (1982) Bovine pancreatic polypeptide-like immunoreactivity in brain and peripheral nervous system: Coexistence with catecholaminergic nerves. Peptides 3: 569590.

Kessler, J. A., W. O. Bell, and I. B. Black (1983) Substance P levels differ in sympathetic target organ terminals and ganglion perikarya. Brain Res. 258: 144-146.

Leblanc, G. G., B. A. Trimmer, and S. C. Landis (1987) Neuropeptide $\mathrm{Y}$-like immunoreactivity in rat cranial parasympathetic neurons: $\mathrm{Co}$ existence with vasoactive intestinal peptide and choline acetyltransferase. Proc. Natl. Acad. Sci. USA 84: 3511-3515.

Lundberg, J. M., and L. Stjärnc (1984) Ncuropeptide y (NPY) depresses the secretion of ${ }^{3} \mathrm{H}$-noradrenaline and the contractile response evoked by field stimulation, in rat vas deferens. Acta Physiol. Scand. 120: $477-479$.

Lundberg, J. M., and K. Tatemoto (1982) Pancreatic polypeptide family (APP, BPP, NPY, and PYY) in relation to sympathetic vasoconstriction resistent to alpha-adrenoceptor blockade. Acta Physiol. Scand. 116: 393-402.

Lundberg, J. M., A. Änggård, J. Fahrenkrug, T. Hökfelt, and V. Mutt (1980) Vasoactive intestinal polypeptide in cholinergic neurons of exocrine glands: Functional significance of coexisting transmitters for vasodilation and secretion. Proc. Natl. Acad. Sci. USA 77: 16511655.

Lundberg, J. M., A. Änggård, P. Emson, J. Fahrenkrug, and T. Hökfelt (1981) Vasoactive intestinal polypeptide and cholinergic mechanisms in cat nasal mucosa: Studies on choline acetyltransferase and release of vasoactive intestinal polypeptide. Proc. Natl. Acad. Sci. USA 78: 5255-5259.

Lundberg, J. M., T. Hökfelt, A. Änggård, L. Terenius, R. Elde, K. Markey, M. Goldstein, and J. Kimmel (1982a) Organizational principles in the peripheral sympathetic nervous system: Subdivision by coexisting peptides (somatostatin-, avian pancreatic polypeptide-, and vasoactive intestinal polypeptide-like, immunoreactive materials). Proc. Natl. Acad. Sci. USA 79: 1303-1307.

Lundberg, J. M., L. Terenius, T. Hökfelt, C. R. Martling, K. Tatemoto, V. Mutt, J. Polak, S. Bloom, and M. Goldstein (1982b) Neuropeptide Y (NPY)-like immunoreactivity in peripheral noradrenergic neurons and effects of NPY on sympathetic function. Acta Physiol. Scand. 116: 477-480.

Lundberg, J. M., L. Terenius, T. Hökfelt, and M. Goldstein (1983) High levels of neuropeptide $Y$ in various mammals including man. Neurosci. Lett. 42: 167-172.

Lundberg, J. M., J. Fahrenkrug, T. Hökfelt, C.-R. Martling, O. Larsson, K. Tatemoto, and A. Änggård (1984a) Co-existence of peptide HI (PHI) and VIP in nerves regulating smooth muscle tone in various mammals including man. Peptides 5: 593-606.

Lundberg, J. M., X.-Y. Hua, and A. Franco-Cereceda (1984b) Effects of neuropeptide $Y$ (NPY) on mechanical activity and neurotransmission in the heart, vas deferens and urinary bladder of the guineapig. Acta Physiol. Scand. 121: 325-332.

Lundberg, J. M., L. Terenius, T. Hökfelt, and K. Tatemoto (1984c) Comparative immunohistochemical and biochemical analysis of pancreatic polypeptide-like peptides with special reference to the presence of neuropeptide $\mathrm{Y}$ in central and peripheral neurons. J. Neurosci. 4: 2376-2386.

Lundberg, J. M., A. Änggård, J. Pernow, and T. Hökfelt (1985) Neuropeptide $Y_{-}$, substance $P_{-}$and VIP-immunoreactive nerves in cat spleen in relation to autonomic vascular and volume control. Cell Tissue Res. 239: 9-18.

Matthew, W. D., L. Tsavaler, and L. F. Reichardt (1981) Identification of synaptic vesicle-specific membrane protein with a wide distribution in neuronal and neurosecretory tissue. J. Cell Biol. 91: 257-269.

McDonald, J. K., M. D. Lumpkin, W. K. Samson, and S. M. McCann (1985) Neuropeptide Y affects secretion of luteinizing hormone and growth hormone in ovariectomized rats. Proc. Natl. Acad. Sci. USA 82: $561-564$.

Moltz, J. H., and J. K. McDonald (1985) Neuropeptide Y: Direct and indirect action on insulin secretion in the rat. Peptides 6: 1155-1159.

Nikkinen, A., J. I. Lehtosalo, H. Uusitalo, A. Palkama, and P. Panula (1984) The lacrimal glands of the rat and the guinea pig are innervated by nerve fibers containing immunoreactivities for substance $P$ and vasoactive intestinal polypeptide. Histochemistry $81: 23-27$.

Ohhashi, T., and D. M. Jacobowitz (1983) The effects of pancreatic polypeptides and neuropeptide $\mathrm{Y}$ on the rat vas deferens. Peptide 4 : $381-386$.

Papka, R. E., J. P. Cotton, and H. H. Taurig (1985) Comparative 
distribution of neuropeptide tyrosine-, vasoactive intestinal polypeptide-, substance P-immunoreactive, acetylcholinesterase-positive and noradrenergic nerves in the reproductive tract of the female rat. Cell Tissue Res. 242: 475-490.

Pernow, J., A. Saria, and J. M. Lundberg (1986) Mechanisms underlying pre- and post-junctional effects of neuropeptide $Y$ in sympathetic vascular control. Acta Physiol. Scand. 126: 239-249.

Ruskell, G. L. (1971) The distribution of autonomic post-ganglionic nerve fibres to the lacrimal gland in monkeys. J. Anat. 109: 229-242.

Said, S. I., and V. Mutt (1970) Polypeptide with broad biological activity: Isolation from small intestine. Science 169: 1217-1218.

Sharkey, K. A., and D. Templeton (1984) Substance P in the rat parotid gland: Evidence for a dual origin from the otic and trigeminal ganglia. Brain Res. 304: 392-396.

Shimizu, T., and N. Taira (1979) Assessment of the effects of vasoactive intestinal peptide (VIP) on blood flow through and salivation of the dog salivary gland in comparison with those of secretin, glucagon, and acetylcholine. Br. J. Pharmacol. 65: 683-687.

Sternini, C., and N. Brecha (1985) Distribution and colocalization of neuropeptide $\mathrm{Y}$ - and tyrosine hydroxylase-like immunoreactivity in the guinea-pig heart. Cell Tissue Res. 241: 93-102.

Stjernquist, M., P. Emson, C. Owman, N.-O. Sjöberg, F. Sundler, and K. Tatemoto (1983) Neuropeptide $Y$ in the female reproductive tract of the rat. Distribution of nerve fibres and motor effects. Neurosci. Lett. 39: 279-284.

Tatemoto, K. (1982) Neuropeptide Y: Complete amino acid sequence of the brain peptide. Proc. Natl. Acad. Sci. USA 79: 5485-5489.

Tatemoto, K., M. Carlquist, and V. Mutt (1982) Neuropeptide YA novel brain peptide with structural similarities to peptide $Y Y$ and pancreatic polypeptide. Nature 296: 659-660.

Terenghi, G., J. M. Polak, J. M. Allen, S. Q. Zhang, W. G. Unger, and S. R. Bloom (1983) Neuropeptide Y-immunoreactive nerves in the uvea of guinea pig and rat. Neurosci. Lett. 42: 33-38.

Thibault, J., D. Vidal, and F. Gros (1981) In vitro translation of mRNA from rat pheochromocytoma tumors, characterization of tyrosine hydroxylase. Biochem. Biophys. Res. Commun. 99: 960-968.

Uddman, R., J. Alumets, B. Ehinger, R. Håkanson, I. Lorén, and F. Sundler (1980a) Vasoactive intestinal peptide nerves in ocular and orbital structures of the cat. Invest. Ophthalmol. Vis. Sci. 19: 878885 .

Uddman, R., J. Fahrenkrug, L. Malm, J. Alumets, R. Håkanson, and F. Sundler (1980b) Neuronal VIP in salivary glands: Distribution and release. Acta Physiol. Scand. 110: 31-38.

Uddman, R., L. Malm, and F. Sundler (1980c) The origin of vasoactive intestinal polypeptide (VIP) nerves in the feline nasal mucosa. Acta Otolaryngol. 89: 152-156.

Warwick, R., and P. L. Williams, eds. (1973) Grays's Anatomy, Saunders, Philadelphia.

Zhang, S. Q., G. Terenghi, W. G. Unger, K. W. Ennis, and J. Polak (1984) Changes in substance $P$-and neuropeptide $Y$-immunoreactive fibres in rat and guinea pig irides following unilateral sympathectomy. Exp. Eye Res. 39: 365-372. 